\title{
Korpuslexikographie und Flexionsmorphologie
}

\author{
Farø, Ken Joensen
}

Published in:

Symposium on Lexicography XI. Proceedings of the Eleventh International Symposium on Lexicography May 24,2002 at the University of Copenhagen

Publication date:

2005

Document version

Også kaldet Forlagets PDF

Citation for published version (APA):

Farø, K. J. (2005). Korpuslexikographie und Flexionsmorphologie. In H. Gottlieb, J. Erik Mogensen, \& A.

Zettersten (Eds.), Symposium on Lexicography XI. Proceedings of the Eleventh International Symposium on Lexicography May 2-4, 2002 at the University of Copenhagen (pp. 217-226). Max Niemeyer Verlag. 
Korpuslexikographie und Flexionsmorphologie

1. Einleitung

Die Angaben zur Flexionsmorphologie gehören zu den weniger beachteten Informationskategorien in der Lexikographie, ${ }^{1}$ sowohl vom Benutzer- als auch vom metalexikographischen Gesichtspunkt her (vgl. Mugdan 1989: 519). In den relativ wenigen Fallen, wo die Flexionsmorphologie im Mittelpunkt einer lexikographischen Untersuchung steht, wie z.B. in Mugdan (1983), wird, von seltenen Ansätzen abgesehen (op.cit:: 203 und 208f.), meist dem Verhältnis zwischen lexikographischer Behandlung und sprachlicher Empirie wenig Aufmerksamkeit zuteil. Die Flexionsparadigmen werden im Allgemeinen als etwas schon Gegebenes betrachtet, ${ }^{2} \mathrm{ob}$ dies nun mit Normtreue zusammenhăngt oder womöglich andere Grïnde hat (siehe u.a. Fahl et al. 2002: 228ff.). ${ }^{3}$

Vor dem Hinterorund der Arbeit mit der Flexionsmorphologie im neuen korpusbasierten "Dänischen Wörterbuch"4 (Den Danske Ordbog, abgekürzt: DDO) der Dänischen Sprachund Literaturgesellschaft (Det Danske Sprog- og Litteraturselskab, abgeklirzt: DSL) möchte ich hier einige Schwierigkeiten beschreiben, die mit der Herausarbeitung der Flexionsangaben in einem Wörterbuch dieses Typus verbunden sind. Und zwar werden Probleme behandelt, die sich in einem Konfliktgebiet befinden, das aus Empirie, Norm und lexikobehandelt, die sich in einem Konfliktgebischen Ansprüchen gebildet wird.

Das Problem kann in điesem Rahmen nur ansatzweise beschrieben werden, obwohl es, da sich ja die korpuslinguistische $e^{5}$ Methode in der lexikographischen Praxis immer mehr verbreitet, eine viel umfassendere Untersuchung verdient hatte.

Vorgesehen ist jedoch ein Artikel mit dem Titel „Lexicography" im geplanten HSK 17 (Morpholo-

gie), Bd. 2 (siehe Literaturverzeichnis: Booij et al.).
Eine wichtige Ausnahme davon bildet Karlsson (2000), der sich jedoch leider nicht mit den Konsequenzen für die Lexikographie beschaftigt (siehe unten).

Trap-Jensen (2002), der aus Grïnden der Editionspraxis zwar vor diesem Artikel erschienen ist, jedoch spater vorgelegt wurde, beschaftigt sich ebenfalls mit Problemen der lexikographischen Norm und Empirie, wenn auch in einem weiteren Kontext, indem sowohl Orthographie, Bedeutungen, Lemmaselektion und Flexion einbezogen werden

Als sechsbandiger moderner Nachfolger des 28-bändigen "Ordbog over det Danske Sprog" aus den Jahren 1919-1956 konzipiert

Zur Kritik dieses Terminus: siehe Bergenholtz (1996: 7f.). 
2. DDO und seine Flexionsangaben

"In comparison to other grammatical characteristics, corpus-based analyses of morphological characteristics are relatively straightforward" (Biber et al. 1998: 58). Dies ist eine Haltung, der man in der korpuslinguistischen Literatur ofters begegnet.

In der Tat schien die Aufgabe relativ unkompliziert zu sein: die Flexion der Lemmat des Dănischen Wörterbuchs zu beschreiben. Dabei sollten zwei verschiedene Quellen als Hauptgrundlage dienen: teils die Empirie, die aus dem 40 Millionen Textwörter umfassenden Textkorpus des Worterbuchs besteht (siehe unten), teils die Angaben des offiziellen dänischen Rechtschreibwörterbuchs, das vom dänischen Sprachrat herausgegeben wird (siehe Dansk Sprognæevn 2001), einer Instanz, die u.a. die für die dänischen Behörden und Schulen verbindliche Orthographie festlegt. Diese Quellenheterogenität rührt vom Wunsch her, das Wörterbuch nicht bloß als eine wissenschaftlich interessante Dokumentation de zeitgenössischen dänischen Lexik zu konzipieren, sondern gleichzeitig auch als ein Werkzeug zur Sprachproduktion, womit đer Wert des Wörterbuchs für die Öffentlichkeit erhöht werden sollte (Norling-Christensen/Asmussen 1998: 224) - schlieBlich wurde das Projekt zur Hälfte von offentlichen Mitteln getragen.

Wo Diskrepanzen zwischen den beiden Quellentypen registriert würden, sollten die normwidrigen Formen, falls im Korpus in signifikanter Menge auftretend, im Wörterbuch ebenfalls beschrieben werden, jedoch mit dem Vermerk, dass sie vom Sprachrat nicht sanktioniert sind - ein in der dänischen Lexikographie bisher nicht völlig unbekanntes Phänomen (siehe unten).

Im Folgenden werden - nach Typen aufgeteilt - Beispiele solcher inoffiziellen, im Sprachgebrauch aber frequenten, Formen angefuhrt:

\section{Pluralflexive:}

albums (-mer/-Ø im offiziellen Rechtschreibwörterbuch und anderswo - Korpusbelege: 71 (albums) gegentuber 3 und 9)

baguettes (- $r$ im offiziellen Rechtschreibwörterbuch und anderswo - Korpusbelege: 4 (baguettes) gegenüber 2; Google-Belege: 252 (baguettes) gegenüber 138)

bjørnekloer (Pflanze) (bjørneklo/bjørnekloer im offiziellen Rechtschreibwörterbuch und anderswo - Korpusbelege: 3 (bjornekløer) gegenaber 0 und 0; Google-Belege: ca. 25 (bjørnekløer) gegenuber (?) und 12) (Sg.: bjørneklo)

champignons (-er im offiziellen Rechtschreibwörterbuch und anderswo - Korpusbelege: 33 (champignons) gegenuber 172)

\section{Determinativflexive:}

balsamen (balsammen im offiziellen Rechtschreibwörterbuch und anderswo) - Korpusbelege: 1 (balsamen) gegenüber 0; Google-Belege: 97 (balsamen) gegenuber 14) bonusen (bonussen im offiziellen Rechtschreibwörterbuch und anderswo - Korpusbelege: 3 (bonusen) gegenuber 1; Google-Belege: 413 (bonusen) gegenüber 330

Die beiden bei weitem umfangreichsten Kategorien inoffizieller Flexion bei den Substantiven im Korpus sind:

a) Fremdwörter aus dem Englischen und aus den romanischen Sprachen, die in der Empirie ein $-s$ im Plural haben, was vom Dänischen Sprachrat in vielen Fallen nicht anerkannt wird (etwa $50 \%$ der Fälle)

und

b) Wörter, davon auch einige fremde, die bis vor kurzem, d.h. bis zur dritten Ausgabe des Rechtschreibwörterbuchs (Dansk Sprognæevn 2001), in ihren deklinierten Formen normgerecht ohne Konsonantengemination geschrieben werden konnten, was in der Empirie tatsăchlich sehr häufig vorkommt. Mit der neuen Ausgabe des offiziellen orthographischen Worterbuchs wurden diese Formen in der Norm abgeschafft. Damit wurden auf einmal sehr viele Flexive inoffiziell. Einerseits will Das Dänische Wörterbuch zwar darauf aufmerksam machen, doch andererseits sollte auch der Sprachgebrauch beschrieben werden, der im Korpus zu finden ist, was die primäre Aufgabe des Wörterbuchprojekts ist (ungefahr $33 \%$ aller Fälle).

Fălle wie die oben dargestellten konnten meistens ohne große Schwierigkeiten registriert und beschrieben werden.

Das Verfahren, inoffizielle Formen ins Wörterbuch aufzunehmen, ist nicht einzigartig in der dănischen Lexikographie. So haben mittlerweile sowohl das Bedeutungs- als auch das Rechtschreibwörterbuch aus dem Verlag Politiken (= Becker-Christensen et al. 1999 bzw. Appel et al. 2001) häufig vorkormmende inoffizielle Formen kodifiziert und diese als solche markiert. Doch hier scheint es sich ausschließlich um orthographische und nicht um flexionsmorphologische Probleme zu handeln. Außerdem liegt dem Verfahren dieser Worterbücher, vom dänischen Sprachrat nicht sanktionierte Wortformen zu lemmatisieren, offenbar der Wunsch zugrunde, dem Benutzer zu helfen, die orthographisch normierte Form im Wörterbuch überhaupt zu finden, falls er bloß eine (frequente) "unkorrekte" Form kennt (siehe op. cit., Vorwort S. 7 bzw. 8).

3. Das Verhältnis zur offiziellen Rechtschreibung

Das Dănische Worterbuch ist nicht in erster Linie ein Rechtschreibwörterbuch - deren gibt es im Dänischen mittlerweile vier, was zu gewissen prinzipiellen Kompetenzproblemen führen kann - und tatsächlich fulhrt (siehe Fahl et al. 2002). Weil aber das Wörterbuch auch als Werkzeug der normgerechten Sprachproduktion dienen soll, so wird die offizielle Rechtschreibung immer berulcksichtigt, wenn auch nicht in jedem Fall kritiklos 
ubernommen - zumindest nicht auf dem Gebiet der Flexionsmorphologie. Wenn das Wö̈r offiziellen dănischen Rechtschreibung folgt, so gilt dies mit gewissen Worterbuch somit Hauptziel des Worterbuchs zusammenhängen, den KernEinschränkungen, die mit dem. Hauptziel des Worts

Die Einschränkungen sind folgende:

a) Das Wörterbuch enthält Wörter, vor allem neuere, die vom offiziellen dänischen Rechtschreibwörterbuch, aus welchen Grunden auch immer, (noch?) nicht behandelt worden

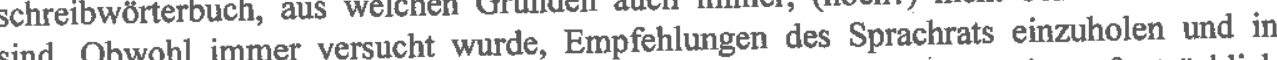
sind. Obwohl immer versucht wurde, Empfehlungen des sprachrats einzuhola auf tatsachlich einigen Fällen auch Analogieschlusse zu ziehen, vor allon beschriebene Simplizia im Rechtschreibwörterbuch, hat die Flexion dieser Worter Prinzip keinen offiziellen Status, solange diese nicht im orthographischen Worterbuch des Sprachrats kodifiziert sind. Dies ist ein prinzipielles Problem und hätte vielleicht im DDO expliziter gehandhabt werden können. Jedenfalls ist die jetzige Situation die, dass im Daexpliziter gehandhabt werdenive als "uofficielt" (,inoffiziell') markiert werden, die zu den nischen Worterbuch nur Fibrobuch gegebenen Formen in expliziter Opposition stehen.

b) b) Das andere Problem betriff Worter, dich verifizier dort aber Flexionsparadigmen besitzen, die 6 , the theoretically possible word forms werden konnten - das, was Karlsson (2000: 647) "he theoretically $(1998: 324)$ meint nennt (siehe auch Fahl et al. 2002: 228ff.). Obwohl Carstairs-McCarthy (1998. 324) meint dass ,paradigm inconsistency never, or almost never arises", so haben die Erfahrungen aus dem DDO-Projekt gezeigt, dass dies eine zu schemenhafte Verallgemeinerung ist, die von dem DDO-Projekt gezeigt, dass derden kann. In der Tat trifft zu, was Karlsson (2000: 652) der Korpusarbit nicht be a gradient phenomenon".

treffend formuliert hat: „Defectivity turns out to be a gradient phenomench Im Korpus des DDO gibt es somit eine Menge von Wörtern, die entweder das Phänomen der „absolute defectivity" (aufgrund gram Gründen von „relative defectivity“ geprăgt sind eher semantischen und pragmatischen Grunden von "relative dnstanz in nicht wenigen (Karlsson 2000: 651) und die gleichzeitig von der normierenden Instanz in nicht weteilt beFällen „mechanisch“ ein rein grammatisch legitimiertes Flexionsparadigma zugeteilt be(a) kommen haben. Diese werden im Dauch dem Interpreten zu sonderbar erscheint. Dadurch Korpus nie im Plural vorkommt und auch dem Interpretreibung näher gekommen zu sein. In glauben wir der Zielsetzung der Empirie-treuen Beschreibung năher gekoren Wörterbüchern manchen Fallen ist sogar das ganze Paradigma, das in den traditionelleren Worterbuchern angegeben wird, aufgrund von Korpusanalysen

gebrauch nur unflektiert vorzukommen scheint.

Diese Reduktionen absolut und relativ defektiver Paradigmen stellen übrigens wohl kaum eine Einschränkung der Benutzerfreundlichkeit des Wörterbuchs als Textproduk-

6 Twar hat mit der neuen Ausgabe des Rechtschreibwörterbuchs (Dansk Sprognævn 2001) die Tlexionsmorphologie des Wőrterbuchs einen Schritt in Richtung einer mehr empirischen Beschreibung gemacht. Doch gleichzeitig ist dadurch, weil diese Neuerung keineswegs konsistent durchgefuhrt ist (siehe Fahl et al. 2002: 228ff.), das Gesamtbild heterogener geworden. Und eine wichtige Fro (ist in diesem Zusammenhang natürlich auch, in welc buch sich überhaupt nach empirischen Daten richten sollte. tions-werkzeug dar, denn der Benutzer braucht die ausgelassenen Formen sicherlich gar nicht.

4. Probleme der praktischen Umsetzung

Im Folgenden werden ein paar Beispiele für die Schwierigkeiten gezeigt, die während der Arbeit auftauchten. Es handelt sich um die Korpusgröße, sowie um das Problem der sondersprachlichen Flexive.

\subsection{Zum Problem der Korpusgröße}

Dass zur Lősung verschiedener lexikographischen Probleme unterschiedliche Korpusgrößen erforderlich sind, wurde schon mehrmals in der Forschung erwähnt (siehe z.B. Bergenholtz 1996: 7). Doch wie groß muss ein Korpus sein, um eine adäquate Grundlage für die Beschreibung der Flexionsmorphologie eines Wörterbuchs zu bilden? Diese Frage ist nur pauschal beantwortbar. Erstens hängt ihre Antwort von der Größe des zu beschreibenden Sprachausschnitts ab, zweitens ist sie vom Ehrgeiz der Herausgeber abhängig: Wie detailliert mochte man die Flexionsmorphologie beschreiben?

Das Korpus des Dänischen Wörterbuchs besteht aus 40 Mio. Textwörtern. Auf der Grundlage von mehreren Untersuchungen wurde dies als hinreichend betrachtet, um den Kernwortschatz des Gegenwartsdânischen unter seinen wesentlichsten semantischen, morphologischen, syntaktischen und phraseologischen (u.a.m.) Aspekten relativ grundlich zu beschreiben.

Von einem so genannten "totalsprachlichen" Wörterbuch, das heißt eines, das auch Fachsprachen decken sollte, wurde in einer anderen Untersuchung von 50 Mio. Textwörtern ausgegangen (siehe Bergenholtz 1996: 9),

$\mathrm{Ob}$ diese Zahlen nun für sămtliche lexikographischen Detailaufgaben hinreichend sind, kann sicherlich diskutiert werden. Obwohl im Korpus für die allermeisten Untersuchungen reichliche, ja ubberwaltigende Mengen an Material zur Verfugung stehen, gibt es einzelne Problembereiche, für die externe empirische Quellen einbezogen werden mussten, wenn die Grundlage der Entscheidungen nicht Introspektion oder nonempirische Quellen sein sollte. Die Flexionsmorphologie gehört zu diesen Bereichen.

Zwar ist es in den meisten Fällen möglich, die Flexionsmorphologie aufgrund der Korpusdaten zufriedenstellend zu beschreiben. Doch vor allem bei Wörtern, die zu den weniger frequenten im Korpus gehören, besteht das Problem, dass z.B. im Korpus keine Pluralformen vorkommen. In einigen Fällen ist sogar überhaupt kein Flexionsparadigma vorhanden. Manchmal hängt dies wirklich mit dem Sprachgebrauch zusammen, wo das Wort nur im Singular oder immer gänzlich unflektiert vorkommt. In anderen Fällen aber wird bei der Zuhilfenahme zusătzlicher empirischer Quellen deutlich, dass die Datenlage des Korpus in diesem Punkt einfach nicht groß genug ist, und dass die Formen durchaus in der Allgemeinsprache im Gebrauch sind. 
Natürlich kann man kritisieren, dass externe Quellen in die Analyse Eingang finden, denn in diesem Punkt wird ja damit die Operationalisierbarkeit der Arbeit angetastet, ein Dilemma, das in der Korpuslinguistik zu den altbekannten Problemen gehört. Man sollte aber stets vor Augen haben, was in solchen Ausnahmefallen wichtiger ist: die methodologische Konsequenz, der zuliebe man auf weitere Untersuchungen verzichtet und somit dem Benutzer Details verschweigt, oder aber die empirische Sprachbeschreibung an sich Wie auch immer sie durchgeführt wird.

\subsection{Zum Problem der sondersprachlichen Flexive}

Wie verfährt man in den Făllen, in denen eine Flexionsform in allgemeinsprachlichen Texten nicht vorkommt, weil die Flexionsform als fachsprachlich anzusehen ist? Dies ist ein Problem, das für die meisten Wörterbuchtypen von Belang ist, nicht nur für da Korpuswörterbuch, obwohl es in diesem Fall ohne Zweifel deutlicher zu Tage tritt, als in den traditionelleren Wörterbüchern. Mugdan (1983: 208f.) hat gezeigt, dass in der Wörterbuchpraxis auf diesem Gebiet große Heterogenităt herrscht. Dies ist immer noch feststellbar, zumindest in der dänischen und deutschen Lexikographie. Vor allem bei so genannten Stoffbezeichnungen" (siehe z.B. Helbig/Buscha 1991: 229) wird man mit dem Problem "konfrontiert, wie beispielsweise bei den Wörtern beton und cement (deutsch: Beton und Zement, siehe unten). In der neuesten Ausgabe des offiziellen dänischen Rechtschreibwörterbuchs (Dansk Sprognævn 2001) haben diese beiden Wörter ein volles Flexionsparadigma. Dies ist auch in sämtlichen konkurrierenden orthographischen Wörterbüchern (Appel et al. 2001, Axelsen et al. 2001, und Bergenholtz et al. 1999), sowie im großen Bedeutungswörterbuch (Becker-Christensen 1999) der Fall. Doch ist diese Situation so selbstverständlich? Man findet den Plural im Korpus nicht und in anderen empirischen Quellen nur in genuinen Fachtexten wie den folgenden, die im Internet gefunden wurden:

\section{beton ('Beton'):}

Hardeegenskaber af cementfattige betoner - effekter ved delvis substitution af cement med mikrosilica og flyveaske

www.civil.auc.dk/i6/forskpr/materials.html

Disse betoner er ikke underlagt DBC. Der anvendes sand i materialeklasse A, sten i materialeklasse $M$ samt dansk cement og puzzolaner

www.bb-beton.dk/betonpris.htm

\section{cement ('Zement'):}

Bro Cement kombinerer fordelene ved' agte glasionomer og resinbaserede cementer. Glasionomerdelen giver fluorfrigørelse og kemisk binding til tandsubstans

www.3m.com/market/dental/dme/dk/prod_html/products/dk_prod_krone.html
Hurtighardnende cementer (styrkeklasse 52,5) kan anvendes til vinterbyggeri, hvor en hurtig hardning af mortlen har betydning

www.mur-tag.dk/muc/materialer/Cement.MLB.htm

Aus diesem Grund hat die 1955-Ausgabe des dänischen Rechtschreibwörterbuchs wohl auch bei beton die Angabe: ohne Plural.

Hat eine veränderte empirische Lage dazu gefuhrt, dass der Sprachrat seine Beschreiuung ebenfalls verändert hat - seiit der 1986-Ausgabe (Dansk Sprognzvn 1992)? Wahrscheinlich nicht, jedoch hat man inzwischen sicherlich Belege für diesen Plural gefunden. Doch um welche Art von Plural handelt es sich? In der Literatur (z.B. in Mugdan 1983: 208) wird sie manchmal unter der Bezeichnung "Sortenplurale" gefuhrt".

Im Vergleich zur bisher ziemlich homogenen Behandlung in der dänischen Lexikoraphie gibt es in der deutschen divergierende Haltungen dazu, wie man diese Plurale handhaben soll. Im Duden Universalwörterbuch wird die Pluralform zwar gegeben, jedoch mit der Präzisierung, dass es sich um "Arten" handelt (Klosa et al. 2001).

Im Wahrig Deutsches Wörterbuch steht wie in der älteren Ausgabe des dănischen Rechtschreibwörterbuchs "ohne Plural" (Wahrig-Burfeind et al. 2000).

Es gibt in der lexikographischen Praxis somit mindestens vier verschiedene Verfahrensweisen, mit diesem Problem umzugehen, und zwar:

a) die Pluralform wird unkommentiert gegeben

b) die Pluralform wird kommentiert gegeben

c) die Pluralform wird unkommentiert ausgelassen

d) die Pluralform wird kommentiert ausgelassen

Was ist nun am adäquatesten? Diese Frage kann wohl nur relativ zur Hauptfunktion des Wörterbuchs beantwortet werden. Handelt es sich um ein Wörterbuch, das primär Hilfe bei der Textproduktion geben möchte, dann kann man im Prinzip fur ein maximierendes Paradigma argumentieren, d.h. hier kann der Plural gegeben werden. Allerdings würde dann die Hinzufügung von Nutzen sein, dass es sich um keine gewöhnliche Pluralfunktion handelt, sondern um eine, die "Arten" indiziert und die außerdem fachsprachlich ist. Handelt es sich aber um ein dokumentierendes worterbuch, das nur den Kernwortschatz und die Kernbedeutungen dieser Worter beschreiben kann oder will, dann sind diese Pluralformen nicht besonders relevant. Genauso wie von einem Wörterbuch des Kernwortschatzes nicht gefordert werden kann, dass es sămtliche Bedeutungen eines Wortes beschreibt, so kann man auch nicht fordern, dass es Flexionsparadigmen berücksichtigen soll, die in der Allgemeinsprache nicht vorkommen.

Man sollte sich dessen bewusst sein, dass man sich mit Randformen und -bedeutungen befasst, wenn man diese fachsprachlichen Plurale in einem gemeinsprachlichen Wörterbuch

An dieser Stelle soll auch auf den Artikel Mass and Collection im geplanten 2. Bd. vom HSK 17 (Morphologie) hingewiesen werden (siehe Booij et al. im Literaturverzeichnis). 
berücksichtigt. Wie Mugdan (1983: 208) uber diese peripheren Pluralformen geschrieben hat: "Wer sie aus irgendwelchen Grunden bilden und verwenden mag, sollte diese Freiheit haben". In ein Wörterbuch des Kern- und Allgemeinwortschatzes gehören sie aber kaum.

\section{Zusätzliche Fragen}

Es könnten in diesem Zusammenhang weitere Probleme diskutiert werden, z.B. das Problem des flexionsmorphologischen Verhăltnisses von Simplex und Kompositum in der Korpuslexikographie: Besteht hier ein Abhängigkeitsverhältnis, oder sollte man Simplex und Kompositum als zwei isolierte Lexeme in ihrem eigenen Recht behandeln? Auch das Problem der lexikalischen Paradigmen gehört in diesen Zusammenhang: ist es eine berechtigte Forderung, Bezeichnungen für Schulfächer, Krankheiten, Münzeinheiten usw. flexionsmorphologisch mőglichst gleich zu behandeln, oder sollten hier einzig und allein die Korpusdaten das Sagen haben?

Schließlich sollte die Frage von diachronischen ${ }^{8}$ Veränderungen in der Flexionsmorphologie (morphologische Innovation) und inwiefern diese in die Lexikographie integriert werden bzw. integriert werden soliten, genauer untersucht und intensiver diskutiert werden, Denn obwohl die Flexionsmorphologie im Vergleich zu anderen grammatischen Kategorien generell konservativ ist (Wurzel 1984: 194), so können auch auf diesem Gebiet Schwankungen und eigentliche Wandlungen registriert werden, wovon auch Das Dänische Wörterbuch Zeugnis ablegt.

Wir können auf diese und weitere Fragen, die zum Problemkomplex Korpuslexikographie und Flexionsmorphologie gehören, an dieser Stelle jedoch nicht weiter eingehen. Doch sollte in diesem Zusammenhang betont werden, dass es sich um ein prinzipielles und furr die zukünttige (Korpus-)Lexikographie weiterhin schwieriges Problem handelt, das tiefer gehende Untersuchungen erfordert.

\section{Zusammenfassung}

In mehreren neueren Arbeiten wurde hervorgehoben, dass Monofunktionalităt eines Worterbuchs erstens ein seltenes Phänomen und zweitens auch nicht wünschenswert sei (siehe dazi z.B. Tarp 1995 und Bergenholtz 1997). Dieser Einwand hat gegen die "naive" Auffassung, es bestehe zwischen Form und Funktion eines Wörterbuchs eine 1:1-Beziehung, ohne Zweifel seine Berechtigung. Dennoch muss man vor Augen haben, dass Polyfunktionalităt die konsistente Herausarbeitung der einzelnen Informationskategorien des Worterbuchs erheblich erschwert. Und dies gilt nicht zuletzt fur die Flexionsmorphologie.

\footnotetext{
" Für eine allgemeinere (nicht-lexikographische) Darstellung, siehe jedoch Joseph (1998).
}

Die Fragen und Schwierigkeiten bei der Erarbeitung der Flexionsmorphologie im Rahmen eines Korpuswörterbuchs mit wesentlichen Nebenfunktionen wie z.B. Hilfe für die Sprachproduktion sind vor allem folgende:

- Ist das Korpus groß genug, um auch die Flexion der weniger frequenten Lemmata empirisch zu erfassen? Einiges deutet darauf hin, dass dieses Detailproblem ein noch größeres Korpus erfordert, als man im Allgemeinen glaubt.

- Wie sollte sich das Wörterbuch gegenüber der offizielien Norm verhalten - falls vorhanden - , wenn das von der Norm Beschriebene in der Empirie kaum oder gar nicht vorkommt und gleichzeitig unwahrscheinlich wirkt?

- Wie sollte man das Problem lösen, dass einige Flexive an bestimmte, sondersprachliche Bedeutungen gebunden sind? Sollte man sie auslassen oder mitnehmen, und dies gegebenenfalls dies explizit kommentieren?

- Wie sollte das Verhaltnis zwischen Simplizia und Komposita sein? Sollten diese flexionsmorphologisch völlig getrennt beschrieben werden, oder zieht die Behandlung des Simplex eine bestimmte Beschreibung der Komposita nach sich?

und schließlich:

- Ist die Forderung nach Konsequenz, die in der Metalexikographie, darunter nicht zuletzt in den Rezensionen, oft zu hören ist, eine berechtigte Forderung, wenn die Empirie alles andere als homogen ist?

Auch auf einem relativ leicht überschaulichen und anscheinend objektivierbaren Gebiet wie der Flexionsmorphologie herrscht in einigen Punkten letztendlich eine gewisse Willkür in der Korpuslexikographie. Und dies kann wohl gar nicht anders sein, denn ,there are many unclear and borderline cases" (Karlsson 2000: 649). Trotzdem stellt die Beschreibung der Flexionsmorphologie im Dănischen Wörterbuch hoffentlich einen Fortschritt dar, weil stets die Empirie die Grundlage der Herausarbeitung bildet, wodurch es sich von den herkömmlichen dänischen Wörterbüchern unterscheidet. Diese haben aber auch nicht unbedingt das gleiche Ziel wie Das Dänische Wörterbuch.

Will man auf dem Gebiet der Flexionsmorphologie Konsequenz im strengsten Sinne des Wortes, dann muss man sie schaffen indem man ein Wörterbuch ohne Rücksicht auf den Sprachgebrauch konstruiert. Die Empirie kann sie nicht liefern. Denn die Sprache ist bel weitem nicht immer so logisch strukturiert, wie es für die Linguisten wünschbar wäre.

Literatur

Appel, Vibeke; Christian Becker-Christensen, Iben Rasmussen et al. (2001): Politikens Retskrivningsordbog. Kopenhagen: Politiken. 
Axelsen, Jens; Grethe Hjorth, Thomas Ingemann, Pia Vater (2001): Retskrivningsordbog. Kopenhagen: Gyldendal.

Becker-Christensen, Christian et al. (1999): Politikens Nudansk Ordbog med etymologi. Kopenhagen: Politiken.

Bergenholtz, Henning (1996): Korpusbaseret leksikografi. In: LexicoNordica 3: 5-17.

- (1997): Polyfunktionale ordbøger. In: LexicoNordica 4: 15-29.

(1999): Danskordbogen. Aarhus: Systime/Center for Leksikografi.

-; Joachim Mugdan (1979): Einführung in die Morphologie. Stuttgart: Kohlhammer.

Biber, Douglas; Susan Conrad, Randi Reppen (1998): Corpus Linguistics. Investigating Language Structure and Use. Cambridge: Cambridge University Press.

Booij, Geert; Christian Lehmann, Joachim Mugdan et al. (Hg.) (2000): Morphologie. Ein internationales Handbuch zur Flexion und Wortbildung (= HSK, Bd. 17.1). Berlin, New York: de Gruyter. Carstairs-McCarthy, Andrew (1998): Paradigmatic Structure: Inflectional Paradigms and Morphological Classes. In: A. Spencer/A. N. Zwicky (Hg.): 322-334.

- (2000): Lexeme, Word-form, Paradigm. In: G. Booij, C. Lehmann, J. Mugdan (Hg.): 595-606.

Dansk Sprognæun (1980 [1955]): Retskrivningsordbog. Kopenhagen: Gyldendal.

- (1992 [1986]): Retskrivningsordbogen. Kopenhagen: Gyldendal.

- (2001): Retskrivningsordbogen. Kopenhagen: Dansk Sprognevn und Aschehoug.

Fahl, Laurids; Ken Fare, Jan Heegaard, Henrik Lorentzen (2002): Review of: Retskrivningsordbogen, 3. udgave, Dansk Sprognæwn og Aschehoug, 2001, Retskrivningsordbog. Gyldendal, 2001, Politikens Retskrivningsordbog, 1. udgave. In; Danske Studier 99: 222-237.

Helbig, Gerhard und Joachim Buscha (1991): Deutsche Grammatik. Ein Handbuch für den Auslanderunterricht. Berlin: Langenscheidt.

Hjorth, Ebba und Kjeld Kristensen et al. (2003f): Den Danske Ordbog. Kopenhagen: Gyldendal und DSL.

Joseph, Brian D. (1998): Diachronic Morphology. In: A. Spencer/A. N. Zwicky (Hg.): 351-373.

Karlsson, Fred (2000): Defectivity. In; G. Booij, C. Lehmann, J. Mugdan (Hg.): 647-653.

Klosa, Annette; Kathrin Kunkel-Razum, Werner Scholze-Stubenrecht, Matthias Wermke (2001): Duden Deutsches Universalwörterbuch. Mannheim: Duden.

McEnery, Tony; Andrew Wilson (1997) [1996]: Corpus Linguistics. Edinburgh: Edinburgh University Press.

Mugdan, Joachim (1983): Grammatik im Wörterbuch: Flexion. In: Germanistische Linguistik 14/1982: 179-237.

- (1989): Information on Inflectional Morphology in the General Monolingual Dictionary. In: Franz Josef Hausmann, Oskar Reichmann, Herbert Emst Wiegand, Ladislav Zgusta (1989-1991): Wörterbücher. Ein internationales Handbuch zur Lexikographie. Berlin: de Gruyter.

Norling-Christensen, Ole and Jørg Asmussen (1998): The Corpus of the Danish Dictionary. In: Lexikos 8: 223-242.

Spencer, Andrew and Arnold N. Zwicky (Hg.) (1998): The Handbook of Morphology. Oxford: Blackwell Publishers.

Stump, Gregory T, (1998): Inflection. In: A. Spencer/A. N. Zwicky (Hg.): 13-43.

Tarp, Sven (1995): Wörterbuchfunktionen: Utopische und realistische Vorschläge für die bilinguale Lexikographie. In: Germanistische Linguistik 127-128:17-61.

Trap-Jensen, Lars (2002): Descriptive and Normative Aspects of Lexicographic Decision-Making: The Borderline Cases. In: A. Braasch, C. Povlsen (Hg.): Proceedings of the Tenth EURALEX International Congress. Vol. II. Kopenhagen: CST: 503-508.

Wahrig-Burfeind, Renate et al. (2000): Wahrig Deutsches Wörterbuch. Gütersloh/München: Bertelsmann Lexikonverlag

Wurzel, Wolfgang Ullrich (1984): Flexionsmorphologie und Natürlichkeit. Ein Beitrag zur morphologischen Theoriebildung. Berlin: Akademieverlag.

\section{Franziskus Geeb}

Perspektiven der Rechneranwendung in der Lexikographie: Lexikographische Datenmodellierung und Datenstrukturierung in relationalen Datenbanken

1. Vorïberlegungen

\subsection{Perspektiven}

Die Perspektiven der Rechneranwendung in der Lexikographie verstanden als die Facetten der möglichen Technologien sind vielfältig und Gegenstand umfassender Forschung (Lemberg, Schröder \& Storrer 2001). Eine besondere Perspektive der Rechneranwendung ist die Nutzung der hiermit gegebenen Möglichkeiten als Modellierungswerkzeug. ${ }^{1}$ So ist eine Modellierung und damit Begründung von lexikographischen Informationen gerade durch diese Werkzeuge denkbar und bereits vollzogen. ${ }^{2}$ In der Regel werden in der lexikographischen Produktion jedoch neben Texteditoren der verschiedensten Art auf Grund ihrer weiten Verbreitung auch relationale Datenbanken eingesetzt. Diese Datenbanken ermöglichen durch den Entwurfsschritt Entity-Relation-Ship-Diagramm und teilweise durch die Schritte der Normalisierung entscheidende Vortberlegungen zur Konzeption eines lexikographischen Nachschlagewerkes ${ }^{3}$. Die wesentliche Perspektive der Rechneranwendung in der Lexikographie, die im Folgenden diskutiert werden soll, ist damit die Nutzung des Rechners als Hilfe zur Modellierung und Modelldarstellung von lexikographischen Daten und Informationen.

\subsection{Lexikographische Information}

Auf der Grundlage einer linguistischen Zeichendefinition lässt über die lexikographischen Relevanzkriterien (z.B. die Bestimmung des Benutzers in der Benutzermatrix: Geeb 1998 39ff;) eine Fortschreibung von lexikographischen Daten in lexikographische Information vertreten (Geeb 2001, $27 \mathrm{ff}$, Geeb 2002). Die lexikographische Information entsteht damit erst und ausschließ1ich durch die dem Lexikographen bewusste oder auch unbewusste Bin-

Datenmodellierung sei verstanden als der Entwurf von (lexikographischen) Dateneinheiten und ihren funktionalen Beziehungen ohne Rücksichtname auf bereits bestehendes Datenmaterial. Datenmodellierung (in Datenbanken und in Auszeichnungssprachen) ermöglicht dabei einen erheblich höheren Freiheitsgrad als Datenstrukturienung. Datenstrukturierung sei im folgenden verstanden als die Tätigkeit, bei der eine Struktur auf bestehendem (lexikographischen) Material aufgesetzt wird. Hauptkennzeichen der Datenstrukturesung ist damit dio Analyse bestehenden Datenmaterials und dann die folgende Erstellung

Für Auszeichnungssprachen (eXtensible Markup Language) z.B. Geeb 2001.

Geeb 1998, 19 ff; auch: Kunze \& Wagner 2001, 229; Simonsen 2001. 